\title{
Statistical inference based on progressively type II censored data from Weibull model
}

\author{
Raed R. Abu Awwad ${ }^{\mathrm{a}}$, Mohammad Z. Raqab ${ }^{\mathrm{b} *}$, Intesar Al-Mudahakhac \\ ${ }^{a}$ Department of Mathematics, University of Jordan, Amman 11942, Jordan \\ ${ }^{\mathrm{b}, \mathrm{c}}$ Department of Statistics and Operations Research, Kuwait University, P.O. Box 5969 Safat, 13060 \\ Kuwait
}

\begin{abstract}
In this paper, we consider the problem of estimating the shape and scale parameters and predicting the unobserved removed data based on a progressive type II censored sample from the Weibull distribution. Maximum likelihood and Bayesian approaches are used to estimate the scale and shape parameters. The sampling-based method is used to draw Monte Carlo (MC) samples and it has been used to estimate the model parameters and also to predict the removed units in multiple stages of the censored sample. A real data set is presented and analyzed for illustrative purposes and Monte carlo simulations are performed to study the behavior of the proposed methods.
\end{abstract}

Keywords: Maximum likelihood estimation, Bayes estimation, Bayes prediction, Monte Carlo simulation.

\section{Introduction}

In the last few years progressive censoring scheme has received considerable attention in the literature. This scheme allows one to remove experimental units at points other than the terminal point of the experiment. It can be described as follows. Under this general censoring scheme, $n$ units are placed on a life-testing experiment and only $m(<n)$ are completely observed until failure. The censoring occurs progressively in $m$ stages. These $m$ stages offer failure times of the $m$ observed units. At the time of the first failure (the first stage) $X_{1: m: n}, r_{1}$ of the $n-1$ surviving units are randomly removed (censored) from the experiment. Similarly, at the time of the second failure (the second stage) $X_{2: m: n}, r_{2}$ of the $n-2-r_{1}$ surviving units are randomly removed (censored) from the experiment. Finally, at the time of the $m^{\text {th }}$ failure (the $m^{\text {th }}$ stage) $X_{m: m: n}$, all the remaining $r_{m}=n-m-\left(r_{1}+r_{2}+\ldots+r_{m-1}\right)$ surviving units are removed from the experiment. We will refer to this as progressive type II censoring scheme $\left(r_{1}, r_{2}, \ldots, r_{m}\right)$. Notice that this scheme includes the type II censoring scheme $\left(r_{1}=r_{2}=\ldots=r_{m-1}=0, r_{m}=n-m\right)$ and complete sampling scheme $\left(r_{1}=r_{2}=\ldots=r_{m}=0\right)$.

Here, the sampling-based technique is applied to Bayesian inference for estimating the unknown parameters as well as predicting the unobserved removed units based on progressive type-II censored data from Weibull distribution (WE) distribution. The WE distribution with parameters $\alpha$ and $\lambda>0$ has the cumulative distribution function (cdf)

$$
F(x \mid \alpha, \lambda)=1-e^{-\lambda x^{\alpha}}, x>0, \alpha, \lambda>0,
$$

and probability density function (pdf)

$$
f(x \mid \alpha, \lambda)= \begin{cases}\alpha \lambda x^{\alpha-1} e^{-\lambda x^{\alpha}} & \text { if } x>0 \\ 0 & \text { if } x \leq 0\end{cases}
$$


respectively. Here $\alpha>0$ and $\lambda>0$ are the shape and scale parameters. From now on, the WE distribution with the shape and scale parameters $\alpha$ and $\lambda$ will be denoted by $\mathrm{WE}(\alpha, \lambda)$. A detailed discussion of the WE distribution has been provided by Johnson et al. (1995).

Let $X_{1: m: n}, X_{2: m: n}, \ldots, X_{m: m: n}$ denote the above mentioned $m$ progressively type II censored sample with progressive censoring $\left(r_{1}, r_{2}, \ldots, r_{m}\right)$. In recent years, several articles on statistical inference for various distributions under this censoring scheme have appeared. See, for example, $\mathrm{Ng}$ (2005), Balakrishnan and Kateri (2008), Basak et al. (2006), Kim et al. (2011) and Kundu and Raqab (2012). In this current work, we extend the work of Kundu and Raqab (2012) to include the progressive censoring type II data from the two-parameter WE distribution.

\section{Maximum likelihood estimation}

Let $\mathbf{X}=\left(X_{1: m: n}, X_{2: m: n}, \ldots, X_{m: m: n}\right)$ with $X_{1: m: n} \leq X_{2: m: n} \leq \ldots \leq X_{m: m: n}$ denote the progressively type II censored sample of size $m$ from a sample of size $n$ drawn from a $\mathrm{WE}(\alpha, \lambda)$ with cdf and pdf given in (1) and (2). The likelihood function based on a progressive type-II censored sample from $W E(\alpha, \beta)$ is given by

$$
L(\alpha, \lambda \mid \mathbf{x}) \propto \alpha^{m} \lambda^{m}\left(\prod_{i=1}^{m} x_{i: m: n}^{\alpha-1}\right) e^{-\lambda \sum_{i=1}^{m}\left(1+r_{i}\right) x_{i: m: n}^{\alpha}} .
$$

By differentiating the natural logarithm of the likelihood function with respect to $\lambda$ and $\alpha$ and equating the resulting terms to zero, we get

$$
\frac{m}{\alpha}+\sum_{i=1}^{m} \ln x_{i: m: n}-\left(\frac{m}{\sum_{i=1}^{m}\left(1+r_{i}\right) x_{i: m: n}^{\alpha}}\right) \sum_{i=1}^{m}\left(1+r_{i}\right) x_{i: m: n}^{\alpha} \ln x_{i: m: n}=0,
$$

and

$$
\lambda=\frac{m}{\sum_{i=1}^{m}\left(1+r_{i}\right) x_{i: m: n}^{\alpha}} .
$$

The MLE of $\lambda$ can be obtained using the Newton-Raphson method by solving the non-linear Eq.(4). Once the MLE of $\alpha$ is computed, it is substituted into Eq.(5) to obtain the MLE of $\lambda$. For more details about the existence and uniqueness of these MLEs and uniqueness, see Balakrishnan and Kateri (2008).

\section{Sample-based estimation}

In this section, we use sample-based technique to obtain the Bayes estimates (BEs) of $\alpha$ and $\lambda$ based on progressive censoring data. A natural choice for the priors of $\alpha$ and $\lambda$ would be to assume that the two quantities are independent and their densities are of the following forms:

$$
\pi_{1}\left(\lambda \mid a_{0}, b_{0}\right)=\frac{b_{0}^{a_{0}}}{\gamma\left(a_{0}\right)} \lambda^{a_{0}-1} e^{-b_{0} \lambda} \text { and } \pi_{2}(\alpha) \text { is log-concave }
$$

where $a_{0}$ and $b_{0}$ are chosen to reflect the prior knowledge about $\lambda$ and the support of $\alpha$ is $(0, \infty)$. For more details, see Kudnu (2008).

By combining (3) and (6), we obtain the joint posterior of $\alpha$ and $\lambda$ as 
In light of the progressive censoring data, the above posterior model is essentially an updated version of our prior knowledge about $\alpha$ and $\lambda$ which is given in (6). The conditional posterior distribution of $\lambda$ given $\alpha$ (and the data) is $\operatorname{Gamma}\left(a_{0}+m, b_{0}+\sum_{i=1}^{m}\left(1+r_{i}\right) x_{i: m: n}^{\alpha}\right)$, while the conditional posterior distribution of $\alpha$ given the data is

$$
\pi(\alpha \mid \text { data }) \propto \alpha^{m} \prod_{j=1}^{m} x_{j: m: n}^{\alpha-1}\left(b_{0}+\sum_{i=1}^{m}\left(1+r_{i}\right) x_{i: m: n}^{\alpha}\right)^{-\left(a_{0}+m\right)} \pi_{2}(\alpha),
$$

which is log-concave if the prior density $\pi_{2}(\alpha)$ is log-concave.

The sample-based technique using the conditional posterior distributions can be used to obtain the BEs of $\theta=\alpha$ or $\lambda$ under squared error loss function $L_{1}$, absolute error loss function $L_{2}$ and linear-exponential (LINEX) function $L_{3}$.

The BE of any function of $\alpha$ and $\lambda$ ( $\operatorname{say} \theta$ ) under $L_{1}$ is expressed as

$$
\hat{\theta}_{B 1}=E_{\text {posterior }}(\theta \mid \text { data })=\int_{0}^{\infty} \int_{0}^{\infty} \theta \pi(\alpha, \lambda \mid \text { data }) d \alpha d \lambda .
$$

Under $L_{2}$, the $\mathrm{BE} \hat{\theta}_{B 2}$ will be the median of the posterior distribution of $\theta$, i.e.

$$
\hat{\theta}_{B 2}=\operatorname{Med}_{\text {posterior }}(\theta \mid \text { data }) .
$$

Under $L_{3}$, then for any $\nu \neq 0$ the $\mathrm{BE} \hat{\theta}_{B 3}$ of $\theta$ will be

$$
\hat{\theta}_{B 3}=\left[E_{\text {posterior }}\left(\theta^{-\nu} \mid \text { data }\right)\right]^{-\frac{1}{\nu}}=\left[\int_{0}^{\infty} \int_{0}^{\infty} \theta^{-\nu} \pi(\theta \mid \text { data }) d \alpha d \lambda .\right]^{-\frac{1}{\nu}} .
$$

Consequently, we develop simulation procedure to generate samples from the posterior distribution of $\alpha$ and $\lambda$. The MC samples $\left(\alpha_{l}, \lambda_{l}\right)$ are generated using the following algorithm:

- Step 1: Generate $\alpha_{1}$ from the log-concave density function $\pi(\alpha \mid \lambda$, data), Eq.(9), using the method proposed by Devroye(1984).

- Step 2: Using the current $\alpha$ value, sample $\lambda_{1}$ from the posterior density function of $\lambda$ given $\alpha$ and data, which is $G\left(a_{0}+m, b_{0}+\sum_{j=1}^{m},\left(1+r_{j}\right) x_{j: m: n}^{\alpha}\right)$.

- Step 3: Repeat steps 1 and $2 M$ times and obtain MC samples $\left\{\left(\alpha_{i}, \lambda_{i}\right) ; i=1,2, \ldots, M\right\}$.

These resulting samples $\left(\alpha_{l}, \lambda_{l}\right)$ are used to approximate the BEs of the parameters and also to construct the corresponding simulated confidence intervals.

\section{Sample-based prediction}

In many life testing and reliability situations, it is desirable to predict the unobserved or censored observations from the early observed observations in the same sample. In this section, we consider the problem of predicting the censored observations in the progressive censored sample from the WE distribution. Mainly, our interest is in the posterior density of the $k^{\text {th }}$ order statistic $Y_{k: r_{j}}\left(k=1,2, \ldots, r_{j} ; j=1,2, \ldots, m\right)$ based on observed progressive type-II censored sample, $\mathbf{X}=$ $\left(x_{1: m: n}, x_{2: m: n}, \ldots, x_{m: m: n}\right)$ with $x_{1: m: n} \leq x_{2: m: n} \leq \ldots \leq x_{m: m: n}$. The posterior predictive density of $Y_{k: r_{j}}$ given the observed censored data $\mathbf{X}$ is 
Here $f_{Y_{k: r_{j}} \mid \mathbf{X}}\left(y_{k: r_{j}} \mid \alpha, \lambda\right)$ is the conditional density of $Y_{k: r_{j}}$ given $\alpha, \lambda$ and the data $\mathbf{X}=\mathbf{x}$. Using the Markovian property of progressively type-II right censored order statistics (Balakrishnan and Aggrawala, 2000) and the fact that the conditional density of $Y_{k: r_{j}} \mid x_{j: m: n}$ is just the conditional distribution of the $k^{t h}$ order statistic obtained from a sample of size $r_{j}$ from $G(y)=[F(y)-$ $\left.F\left(x_{j: m: n}\right)\right] /\left[1-F\left(x_{j: m: n}\right)\right], y>x_{j: m: n}$, we have

$$
f_{Y_{k: r_{j}} \mid d a t a}\left(y_{k: r_{j}} \mid \alpha, \lambda\right)=c \alpha \lambda \sum_{i=0}^{k-1}(-1)^{k-i-1}\left(\begin{array}{c}
k-1 \\
i
\end{array}\right) y_{k: r_{j}}^{\alpha-1} e^{-\lambda\left(r_{j}-i\right)\left(y_{k: r_{j}}^{\alpha}-x_{j: m: n}^{\alpha}\right)} .
$$

From (7) and (9), the posterior predictive density of $Y_{k: r_{j}}$ given the observed censored data is

$$
\begin{aligned}
p\left(y_{k: r_{j}} \mid \text { data }\right)= & c \alpha \lambda \int_{0}^{\infty} \int_{0}^{\infty} \sum_{i=0}^{k-1}(-1)^{k-i-1}\left(\begin{array}{c}
k-1 \\
i
\end{array}\right) y_{k: r_{j}}^{\alpha-1} \\
& e^{-\lambda\left(r_{j}-i\right)\left(y_{k: r_{j}}^{\alpha}-x_{j: m: n}^{\alpha}\right)} \pi(\alpha, \lambda \mid \text { data }) d \alpha \lambda .
\end{aligned}
$$

Clearly, the form of the posterior predictive density in (10) is not attractable and the computation of the predictive Bayes estimates $E\left(Y_{k: r_{j}} \mid d a t a\right)$ is not an easy task. Consequently, we use the MC samples described in Section 3 to generate samples from the predictive distributions. Under the square error loss function $L_{1}$, the Bayes predictor (BP) of $Y=Y_{k: r_{j}}$ can be obtained as

$$
\hat{Y}_{k: r_{j}}^{B P_{1}}=\frac{c}{M} \sum_{l=1}^{M} \sum_{i=0}^{k-1}(-1)^{k-i-1}\left(\begin{array}{c}
k-1 \\
i
\end{array}\right) e^{-\lambda_{l}\left(i-r_{j}\right) x_{j: m: n}^{\alpha_{l}}} \times \frac{\Gamma\left(\frac{1}{\alpha_{l}}+1, \lambda_{l}\left(r_{j}-i\right) x_{j: m: n}^{\alpha_{l}}\right)}{\lambda_{l}^{\frac{1}{\alpha_{l}}}\left(r_{j}-i\right)^{\frac{1}{\alpha_{l}}+1}},
$$

where

$$
\Gamma(a, c)=\int_{c}^{\infty} x^{a-1} e^{-x} d x, a>0, c>0,
$$

is the incomplete gamma function. Under $L_{2}$, the corresponding sample-based predictor of $Y=$ $Y_{k: r_{j}}$, denoted by $Y_{k: r_{j}}^{B P_{2}}$, is the median of the posterior predictive density of $Y=Y_{k: r_{j}}$, Eq.(11), which is obtained by solving the following equation with respect to $Y_{k: r_{j}}^{B P_{2}}$

$$
\frac{c}{M} \sum_{l=1}^{M} \sum_{i=0}^{k-1}(-1)^{k-i-1}\left(\begin{array}{c}
k-1 \\
i
\end{array}\right) \times \frac{e^{-\lambda_{l}\left(r_{j}-i\right)\left(\hat{Y}_{k: r_{j}}^{B P_{2}}\right)^{\alpha_{l}}-x_{j: m: n}^{\alpha_{l}}}}{r_{j}-i}=\frac{1}{2} .
$$

Similarly, under the LINEX loss function $L_{3}$ with $\nu \neq 0$, the corresponding sample-based predictor $Y_{k: r_{j}}^{B P_{3}}$ of $Y_{k: r_{j}}$ is obtained to be

$$
\hat{Y}_{k: r_{j}}^{B P_{3}}=\left[\frac{c}{M} \sum_{l=1}^{M} \sum_{i=0}^{k-1}(-1)^{k-i-1}\left(\begin{array}{c}
k-1 \\
i
\end{array}\right) e^{-\lambda_{l}\left(i-r_{j}\right) x_{j: m: n}^{\alpha_{l}}} \times \frac{\Gamma\left(1-\frac{a^{*}}{\alpha_{l}}, \lambda_{l}\left(r_{j}-i\right) x_{j: m: n}^{\alpha_{l}}\right)}{\lambda_{l}^{-\frac{a^{*}}{\alpha_{l}}}\left(r_{j}-i\right)^{1-\frac{a^{*}}{\alpha_{l}}}}\right]^{-\frac{1}{a^{*}}} .
$$

Another important aspect of prediction is to construct a two-sided interval for $Y=Y_{k: r_{j}}(k=$ $\left.1,2, \ldots, r_{j} ; j=1,2, \ldots, m\right)$. To obtain prediction bounds on $Y=Y_{k: r_{j}}\left(k=1,2, \ldots, r_{j} ; j=\right.$ $1,2, \ldots, m)$, we need to find the predictive survival function of $Y=Y_{k: r_{j}}$ at any point $y>x_{j: m: n}$. Based on Eq.(9), the survival function of $Y=Y_{k: r_{j}}$ is defined by

$$
S_{Y \mid \text { data }}(y \mid \alpha, \lambda)=c \sum_{i=0}^{k-1}\left[(-1)^{k-i-1}\left(\begin{array}{c}
k-1 \\
i
\end{array}\right) \frac{e^{-\lambda\left(r_{j}-i\right)\left(y^{\alpha}-x_{j: m: n}^{\alpha}\right)}}{r_{j}-i}\right] .
$$

By using the MC samples $\left\{\left(\alpha_{l}, \lambda_{l}\right) ; l=1,2, \ldots, M\right\}$, the simulated estimator for the predictive survival function of $Y=Y_{k \cdot r}$. under $L_{1}, L_{2}$ and $L_{3}$ can be obtained similarly. A $(1-\beta) \%$ simulated 


\section{Data analysis and numerical comparisons}

In this section, we present the analysis of two real data sets using progressively type II censored data from WE distribution and conduct a simulation study to examine the performances of the sample-based estimates and predictors of the removed units of the censored sample.

\subsection{Data analysis}

Example (real data): In this example, we analyze the times to failure of live specimens which were subjected to progressively censored aging tests on XLPE insulated cable models under combined thermal-electrical stresses at $12 \mathrm{kV}$ and $60^{\circ} \mathrm{C}$. Here, live specimens were removed at selected times and/or at the time of breakdowns. The progressively censored sample was taken as follows:

\begin{tabular}{ccccccc}
\hline$i$ & 1 & 2 & 3 & 4 & 5 & 6 \\
\hline$r_{i}$ & 2 & 1 & 3 & 1 & 3 & 2 \\
$y_{i}$ & 445 & 479 & 489 & 607 & 692 & 969 \\
\hline
\end{tabular}

Montanari and Cacciari (1988) presented several methods for estimating the parameters of the WE distribution as an appropriate fitted model for the aging times. Predictions of the lifetime lengths of the removed units in multiple stages of the progressively censored sample, are of natural interest in this context. Using the iteration procedure described in Section 3, we obtained the sample-based estimates under square error loss function $L_{1}$ for the parameters as follows:

$$
\widehat{\alpha}_{B_{1}}=2.6318, \widehat{\lambda}_{B_{1}}=0.9880,
$$

and $95 \%$ confidence intervals for $\alpha$ and $\lambda$, were determined to be respectively, $(2.2266,2.8061)$ and $(0.4010,1.7880)$. The simulated Bayes predictive estimates and $95 \%$ PIs for $Y_{k: r_{j}}$ are presented in Table 2 with $r_{1}=2, r_{2}=1, r_{3}=3$. It is observed from Table 2 that the Bayes PIs under LINEX loss function perform well when compared with the ones under square error loss and absolute error loss functions in terms of the interval length criterion.

Table 2: Prediction values and $95 \%$ PIs for $Y_{k: r_{i}}$

\begin{tabular}{cccccc}
\hline$Y_{k: r_{j}}$ & $L_{1}$ & $L_{2}$ & $L_{3}(\nu=0.1)$ & $L_{3}(\nu=0.5)$ & $L_{3}(\nu=1.0)$ \\
\hline$Y_{1: r_{1}}$ & 807 & 759 & 765 & 751 & 735 \\
& $(463,1426)$ & $(469,1435)$ & $(463,1278)$ & $(463,1226)$ & $(463,1166)$ \\
$Y_{2: r_{1}}$ & 1190 & 1144 & 1132 & 1111 & 1086 \\
& $(620,2028)$ & $(633,1833)$ & $(620,1746)$ & $(620,1663)$ & $(620,1576)$ \\
$Y_{1: r_{2}}$ & 1015 & 955 & 948 & 926 & 899 \\
& $(510,1876)$ & $(519,1963)$ & $(510,1655)$ & $(510,1589)$ & $(510,1517)$ \\
$Y_{1: r_{3}}$ & 744 & 700 & 718 & 709 & 699 \\
& $(499,1234)$ & $(500,1273)$ & $(499,1114)$ & $(499,1075)$ & $(499,1032)$ \\
$Y_{2: r_{3}}$ & 988 & 954 & 953 & 940 & 925 \\
& $(588,1591)$ & $(591,1501)$ & $(588,1399)$ & $(588,1337)$ & $(588,1266)$ \\
$Y_{3: r_{3}}$ & 1310 & 1267 & 1261 & 1244 & 1222 \\
& $(754,2118)$ & $(779,2013)$ & $(752,1815)$ & $(752,1704)$ & $(751,1578)$ \\
\hline & & & & &
\end{tabular}

\section{$5.2 \quad$ Numerical comparisons}

To examine the performances of the sample-based estimates and predictors, we carried out an MC simulation. For a particular $n, m$ and a censoring scheme, we generate a progressive censored sample from the WE distribution with $\alpha=2$ and $\lambda=1$ using the algorithm 
compute the average bias and mean square error (MSE) of the respective estimate. The results are reported but not displayed here. It can be seen, from the numerical results, the BEs perform better than the MLEs, both in terms of bias and MSE.

It can also be noted that the performances of the MLE as well as the BE are sensitive to both sample size and sampling scheme. While the performances of BEs of $\alpha$ are generally close under $L_{1}$ and $L_{3}$, the BEs of $\lambda$ under $L_{2}$ perform better than their counterparts under $L_{1}$. Further, under LINEX loss function $L_{3}$, the corresponding estimates of $\lambda$ compete all other estimates in the most considered cases.

For the computations of predictors, we have obtained the simulated point predictors. In the prediction method (method 1, say), we substitute the MC samples $\left(\alpha_{l}, \lambda_{l}\right), l=1,2, \ldots, M$ into the posterior predictive density of $Y_{k: r_{j}}$ given the observed censored data which is given in (10) and then obtain an approximate predictor of $Y_{k: r_{j}}$. Another suggested method (call it method 2), is to substitute the MC samples into the conditional density (9) and then simulate $Y_{k: r_{j}}^{l}, j=1,2, \ldots, M$ from the conditional distribution of $Y_{k: r_{j}}$ given data and $\alpha_{l}, \lambda_{l}$. The resulting simulated values $Y_{k: r_{j}}^{1}, Y_{k: r_{j}}^{2}, \ldots, Y_{k: r_{j}}^{M}$ will be a simulated sample from the posterior distribution. Based on this simulated sample, it is evident that method 1 is highly efficient relative to method 2 ; the performances of its predictors in the sense of MSE are better than the ones obtained using method 2.

\section{References}

[1] Balakrishnan, N. and Aggrawala, R. (2000). Progessive censoring, theory, methods and applications, Birkhauser, Boston.

[2] Balakrishnan, N. and Kateri, M. (2008). On the maximum likelihood estimation of parameters of Weibull distribution based on complete and censored data, Statistics 8 Probability Letters 78(17), 2971-2975.

[3] Basak, I, Basak, P. and Balakrishnan, N. (2006). On some predictors of times to failure of censored items in progressively censored camples, Computational Statistics and Data Analysis 50, 1313-1337.

[4] Devroye, L. (1984). A simple algorithm for generating random variates with a logconcave density, Computing 33, 247-257.

[5] Johnson, N.L, Kotz, S. and Balakrishnan, N. (1995). Continuous univariate distribution, 2nd edition, Wiley and Sons, New York

[6] Kim, C., Jung, J., and Chung, Y. (2011). Bayesian estimation for the exponentiated Weibull model under type II progressive censoring, Statistical Papers 52(1), 53-70.

[7] Kundu, D. (2008). Bayesian inference and life testing plan for the Weibull distribution in presence of progressive censoring, Technometrics 50, 144-154.

[8] Kundu, D. and Raqab, M. Z. (2012). Bayesian inference and prediction for a typeII censored Weibull distribution, Journal of Statistical Planning 86 Inference 142(1), 41-47.

[9] Montanari, G. C. and Cacciari, M. (1988). Progressively-censored aging tests on XLPE-insulated cabe models, IEEE Transactions on Electrical Insulation 23(3), 365372.

[10] Ng, H. K. T. (2005). Parameter estimation for a modified Weibull distribution for 